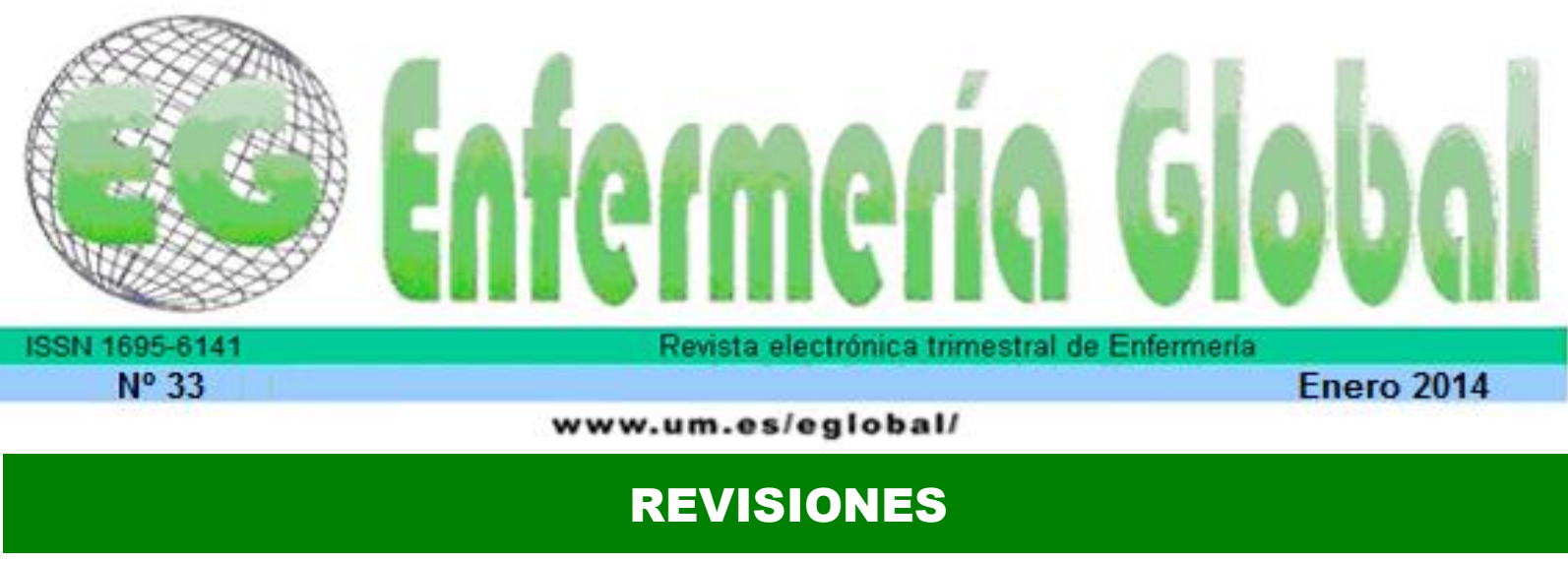

\title{
Cultura de la seguridad del paciente en la atención sanitaria: un estudio bibliométrico
}

Cultura de Segurança do paciente em instituições de saúde: um estudo bibliométrico

Culture of Patient Safety in health care: a bibliometric study

\section{${ }^{*}$ Camargo Tobias, Gabriela ${ }^{* *}$ Queiroz Bezerra, Ana Lúcia ${ }^{* * *}$ Silvestre Branquinho, Nayla Cecília ${ }^{* * * *}$ de Camargo Silva, Ana Elisa Bauer}

\author{
*Alumna de Máster en Enfermería E-mail: gabicamargo22@gmail.com **Doctora en Enfermería. \\ Docente en la Facultad de Enfermería. ${ }^{* * *}$ Máster en Enfermería. ${ }^{* * * * D}$ Doctora en Enfermería. Docente \\ en la Facultad de Enfermería. Universidad Federal de Goiás. Brasil
}

Palabras clave: Seguridad del paciente; gestión de la seguridad: cultura organizacional
Palavras chave: Segurança do paciente; gerenciamento de segurança; cultura organizacional.

Keywords: Patient Safety; security management; organizational culture.

\section{RESUMEN}

El objetivo de este estudio es describir las características de la literatura científica sobre la seguridad de los pacientes en relación con la gestión de la seguridad y la cultura de la organización, el tipo de publicación, año, vehículo de publicación, país, idioma y tema. La recolección de datos se llevó a cabo en junio de 2012, en la Biblioteca Virtual en Salud (BVS), utilizando los descriptores seguridad del paciente, gestión de la seguridad y cultura organizacional. Los resultados evidenciaron 106 indicaciones en siete años (2006 a 2012). 50,9\% de las publicaciones fueron artículos de los Estados Unidos de América, 84,9\% de las publicaciones eran en lengua inglesa, el 74\% eran artículos originales y el tema principal fue la mala praxis. Llegamos a la conclusión de que la producción científica fue mayor y continua en los últimos 3 años. La autoría fue predominantemente estadounidense y publicado en revistas científicas en los Estados Unidos, lo que indica la necesidad de desarrollar la investigación sobre el tema en Brasil.

\section{RESUMO}

O objetivo deste estudo é descrever as características da produção científica sobre segurança do paciente relacionada a gerenciamento de segurança e cultura organizacional, quanto ao tipo de publicação, ano, veículo de publicação, país, idioma e temática abordada. A coleta de dados foi realizada em junho de 2012, na Biblioteca Virtual em Saúde (BVS) utilizando os descritores segurança do paciente, gerenciamento de segurança e cultura organizacional. Os resultados evidenciaram106 indicações num período de sete anos (2006 a 2012). Das publicações 50,9\% foram artigos provenientes dos Estados Unidos da América; 84,9\% publicações foram na língua inglesa; $74 \%$ foram de artigos originais e a principal temática foi o erro médico. Concluímos que a produção científica foi 
maior e contínua nos últimos 3 anos. A autoria foi predominantemente americana e publicada em periódicos científicos nos Estados Unidos, o que indicou a necessidade de desenvolver pesquisas sobre o assunto no Brasil

\section{ABSTRACT}

The objective of this study is to describe the characteristics of scientific literature on patient safety related to security management and organizational culture, the type of publication, year, and vehicle of publication, country, language and theme. Data collection was conducted in June 2012, the Virtual Health Library (VHL) using the keywords patient safety, safety management and organizational culture. The results show 106 indications in seven years (2006 to 2012). 50.9\% of the publications were articles from the United States, $84.9 \%$ publications were in English, 74\% were original articles and the main theme was the malpractice. We conclude that the scientific production was higher and continuous in the last 3 years. The authorship was predominantly American and published in scientific journals in the United States, which indicated the need to develop research on the subject in Brazil.

\section{INTRODUCCIÓN}

La reducción del riesgo de daños innecesarios asociados con la asistencia de salud hasta un mínimo aceptable es una cuestión reconocida a nivel mundial como un componente importante para el logro de la seguridad del paciente y calidad de servicio $^{(1)}$.

En los servicios de salud, la calidad se ha convertido en una ventaja competitiva para las organizaciones hospitalarias. La sociedad exige cada vez más la excelencia en los servicios que a ella son prestados, lo que hace que sea esencial la creación y adecuación en los procesos organizacionales (2) y el desarrollo de una cultura organizacional y de seguridad en la asistencia a los pacientes.

La cultura organizacional es la personalidad de la organización y, como tal, se manifiesta en sus políticas, decisiones y cotidiano de su funcionamiento diario, en las formas con que las personas interaccionan entre sí, cómo hacen su trabajo y comunican entre si $^{(3)}$. Con el análisis de la cultura organizacional de un determinado servicio, es posible identificar cómo es la institución, lo que pretende ser y lo que su gestión es capaz de realizar con sus fuerzas y debilidades para lograr la calidad y desarrollar una cultura centrada en la seguridad del paciente.

En las instituciones de salud, la cultura de la seguridad está emergiendo como uno de los requisitos esenciales para evitar la ocurrencia de incidentes, a través de la gestión del riesgo en el que los profesionales comunican los errores y actúan de forma proactiva en el rediseño de los procesos para evitar nuevos incidentes.

Por lo tanto, si la cultura de la seguridad es importante para una organización y si la organización realiza sus procesos siempre pensando en la seguridad, los resultados positivos serán en beneficio de los pacientes, colaboradores y equipo de salud ${ }^{(4)}$. Hay un consenso de que algunos atributos culturales de la organización contribuyen a la seguridad del paciente, tales como el trabajo en equipo, el soporte de los líderes y la comunicación ${ }^{(5)}$.

Organizaciones con una cultura de seguridad positiva se caracterizan por una comunicación abierta y basada en la confianza mutua, gestión del riesgo, por percepciones comunes acerca de la importancia de la seguridad y el aprendizaje 
organizacional y por la confianza en la eficacia de las medidas preventivas ${ }^{(6)}$. En ese sentido, razón adicional para lograr una cultura de seguridad positiva es el estímulo a la notificación de incidentes por el profesional que es considerada una herramienta eficaz para mejorar la calidad y seguridad del establecimiento ${ }^{(7)}$.

Teniendo en cuenta esto, la utilización de herramientas para evaluación de esa cultura se hace necesaria para comprender las actitudes y comportamientos de los profesionales en relación a la seguridad del paciente. Investigaciones de evaluación deben tener en cuenta la interacción entre factores organizacionales e individuales, lo que permite una mejor comprensión de la dinámica del equipo y de las actitudes individuales en relación a la cultura de seguridad del paciente ${ }^{(8)}$.

Investigar la cultura de seguridad no es sólo identificar la presencia o ausencia de determinadas variables concretas, sino examinar las relaciones individuo-trabajoorganización involucrada en ellas. Esto puede profundizar el conocimiento sobre la cultura de seguridad de una institución con un enfoque en la gestión del riesgos ${ }^{(9)}$. Estas prerrogativas básicas han subsidiado los cambios en favor de la seguridad del paciente en la asistencia sanitaria. Por lo tanto, la seguridad del paciente es considerada como un componente crítico de la calidad de la asistencia.

Por lo tanto, es pertinente y oportuno conocer y reflexionar sobre la seguridad del paciente relacionada con la gestión de la seguridad y la cultura organizacional, ya que esto afecta directamente la práctica profesional. El análisis y diagnóstico, proporcionado por la investigación son herramientas valiosas para conocer lo que se han investigado en ese tema y comprender la evolución de los resultados y soluciones que enfatizan un cuidado seguro para la calidad de la atención.

El presente trabajo tiene como objetivo describir las características de la literatura científica sobre la seguridad de los pacientes en instituciones de salud relacionadas al tipo, año y vehículo de publicación, país, idioma y tema.

\section{MÉTODO}

Estudio descriptivo del tipo bibliométrico, que es un método accesible para el análisis cuantitativo de la producción científica sobre un tema en particular ${ }^{(10)}$. Este método también permite la recuperación de la información, subvenciona la evaluación cualitativa de la actividad científica esencial para que el investigador pueda seguir lo que se produce en su área de estudio ${ }^{(11)}$.

La recolección de datos he sido realizada en junio de 2012, en la Biblioteca Virtual en Salud (BVS), mediante la consulta de la base de datos de la Literatura Latinoamericano y del Caribe de la Salud (LILACS), Medical Literature Analysis and Retrieval System Online (MEDLINE), Índice Bibliográfico Español de Ciencias de la Salud (IBECS), Cochrane Library y Scientific Electronic Library Online (SciELO). Estas bases y bibliotecas fueron elegidas porque tienen gran número de publicaciones en el área de búsqueda. Los descriptores utilizados para la búsqueda, seleccionados de entre los Descriptores en Ciencias de la Salud (DeCS) LILACS y Medical Subject Heading (MeSH) de la MEDLINE, fueron: seguridad del paciente, Administración de la Seguridad y cultura organizacional.

Se encontraron 135 indicaciones y, después del análisis de los registros (título, resumen y descriptores o sólo el título y descriptores), 106 publicaciones fueron consideradas relevantes para el tema discutido. Se utilizó el programa Microsoft Excel 
versión 2007 para construcción de una herramienta de registro de datos, en la que las variables fueron distribuidas, siendo después calculadas las frecuencias simples y relativas.

\section{RESULTADOS}

Se analizaron 106 publicaciones en el período de siete años, 2006 - 2012. La trayectoria de las publicaciones observó tendencia ascendente, desde 2010. En 2011 ocurrió el mayor número de publicaciones 51 (48,1\%), como se muestra en la Tabla 1.

Tabla 1. Publicaciones sobre seguridad del pacientes en las instituciones de salud en el período 2006-2012.

\begin{tabular}{lll}
\hline AÑO & N & $\%$ \\
\hline 2006 & 1 & 0,9 \\
2007 & 1 & 0,9 \\
2008 & 1 & 0,9 \\
2009 & 1 & 0,9 \\
2010 & 14 & 13,2 \\
2011 & 51 & 48,1 \\
2012 & 37 & 34,9 \\
\hline Total & 106 & 100,0 \\
\hline
\end{tabular}

Fueron encontradas indicaciones utilizando los descriptores del tema seguridad del paciente en sólo dos bases de datos, siendo 95 (89,6\%) en el MEDLINE y $11(10,4 \%)$ en la base de datos LILACS. En las bases IBECS, Cochrane y SciELO no fueron encontrados indicaciones.

En cuanto al tipo de estudio, 79 (74,5\%) fueron artículos originales, $14(13,2 \%)$ artículos de revisión, siete $(6,6 \%)$ editoriales, dos $(1,9 \%)$ relatos de experiencia, dos $(1,9 \%)$ noticias, dos $(1,9 \%)$ tesis doctorales, como muestra la Tabla 2.

Tabla 2. Tipos de publicaciones sobre seguridad del paciente en las instituciones de salud en el período 2006-2012.

\begin{tabular}{lll}
\hline Publicaciones & $\mathrm{N}$ & $\%$ \\
\hline Artículo original & 79 & 74,5 \\
Artículo de revisión & 14 & 13,2 \\
Editorial & 7 & 6,6 \\
Relato de experiencia & 2 & 1,9 \\
Tesis & 2 & 1,9 \\
Noticias & 2 & 1,9 \\
\hline Total & 106 & 100,0 \\
\hline
\end{tabular}

La mayor parte de indicaciones ha sido de artículos, lo que explica la superioridad de artículos originales de investigación. En cuanto al vehículo de publicación, 103 títulos $(89,0 \%)$ fueron encontrados en revistas científicas, siete de ellos $(6,6 \%)$ en el 
periódico Journal Patient Safety, cinco (4,7\%) en la revista Quality Safety Health Care, cuatro (3,8\%) en la revista inglesa Revista Calidade Assistance, cuatro $(3,8 \%)$ en la Surgery Clinic North American, cuatro (3,8\%) en la Online Journal y cuatro $(3,8 \%)$ en la International Journal Qualidade Health Care, y las demás contienen entre tres $(2,8 \%)$ y uno $(0,9 \%)$ artículo publicado sobre el tema investigado.

Respecto al idioma utilizado, 90 (84,9\%) publicaciones fueron escritas en Inglés, nueve $(8.5 \%)$ en portugués, siendo dos tesis, cuatro artículos originales, un relato de experiencia, una revisión y un editorial, cuatro $(3,8 \%)$ fueron escritas en español, dos $(1,9 \%)$ en francés y uno $(0,9 \%)$ en alemán.

Tabla 3. Idioma de las Publicaciones sobre seguridad del paciente en instituciones de salud en el período 2006-2012.

\begin{tabular}{lll}
\hline IDIOMA & $\mathrm{N}$ & $\%$ \\
\hline Inglés & 90 & 84,9 \\
Portugués & 9 & 8,5 \\
Español & 4 & 3,8 \\
Francés & 2 & 1,9 \\
Alemán & 1 & 0,9 \\
\hline Total & 106 & 100,0 \\
\hline
\end{tabular}

De los 17 países de origen de las publicaciones, 54 (50,9\%) son de los Estados Unidos de América (EE.UU.), 14 (13,5\%) del Reino Unido, 10 (9,4\%) brasileños, seis $(5,7 \%)$ de los Países Bajos, como muestra la Tabla 4.

Tabla 4. Países de publicaciones sobre seguridad del paciente en las instituciones de salud en el período 2006-2012.

\begin{tabular}{lll}
\hline PAís & $\mathrm{N}$ & $\%$ \\
\hline EE.UU. & 54 & 50,9 \\
Reino Unido & 14 & 13,5 \\
Brasil & 10 & 9,4 \\
Países Bajos & 6 & 5,7 \\
España & 5 & 4,7 \\
Suiza & 4 & 3,8 \\
Francia & 3 & 2,8 \\
Australia & 2 & 1,9 \\
Canadá & 2 & 1,9 \\
Chipre & 1 & 0,9 \\
Finlandia & 1 & 0,9 \\
Italia & 1 & 0,9 \\
Nueva Zelanda & 1 & 0,9 \\
Suecia & 1 & 0,9 \\
Tailandia & 1 & 0,9 \\
\hline Total & 106 & 100,0 \\
\hline
\end{tabular}

La riqueza en la variedad de enfoques del tema puede verse en los datos de la Tabla 5 . 
Tabla 5. Temas abordados sobre seguridad del paciente en instituciones de salud en el período 2006-2012.

\begin{tabular}{|c|c|c|c|}
\hline Temática & & $\mathrm{N}$ & $\%$ \\
\hline Errores Médicos & & 22 & 20,8 \\
\hline Calidad de la atención & & 17 & 16,0 \\
\hline Error en la medicación & & 13 & 12,3 \\
\hline $\begin{array}{l}\text { Administración de } \\
\text { Seguridad }\end{array}$ & la & 13 & 12,3 \\
\hline $\begin{array}{l}\text { Actitud profesional frente } \\
\text { error/riesgo }\end{array}$ & al & 12 & 11,3 \\
\hline Cultura de seguridad & & 9 & 8,5 \\
\hline Indicadores de riesgo & & 4 & 3,8 \\
\hline Liderazgo de enfermería & & 4 & 3,8 \\
\hline Comunicación & & 3 & 2,8 \\
\hline Capacitación en Servicio & & 3 & 2,8 \\
\hline $\begin{array}{l}\text { Recursos humanos } \\
\text { financieros en de salud }\end{array}$ & $y$ & 3 & 2,8 \\
\hline Registro electrónico & & 2 & 1,9 \\
\hline $\begin{array}{l}\text { Normalización } \\
\text { terminología y conceptos }\end{array}$ & de & 1 & 0,9 \\
\hline Total & & 106 & 100,0 \\
\hline
\end{tabular}

Varios temas son evidentes, 22 estudios (20,8\%) abordaron los errores médicos, 17 (16\%) la calidad de la atención, $13(12,3 \%)$, errores de medicación, 13 (12,3\%), gestión de riesgos, $12(11,3 \%)$ la actitud del profesional frente a conductas de seguridad, nueve $(8,5 \%)$ cultura de seguridad, cuatro $(3,8 \%)$ indicadores de riesgo, cuatro $(3,8 \%)$ el liderazgo de enfermería, tres $(28 \%)$ la capacitación en servicio, tres $(2,8 \%)$ comunicación, tres $(2,8 \%)$ recursos humanos y financieros en salud, dos $(1,9 \%)$ registro electrónico y uno $(0,9 \%)$ la normalización de terminologías y conceptos.

\section{DISCUSIÓN}

En las bases de datos consultadas fueron encontradas 106 publicaciones solo desde 2006. Durante tres años consecutivos el número de artículos ha sido escaso, y a partir de 2010 tuvo un aumento significativo de las publicaciones demostrando el interés de los investigadores en el tema. Una de las explicaciones podría ser el hecho de que el mismo año, la Joint Commission International $(\mathrm{JCl})$ ha puesto en marcha las metas internacionales para la seguridad del paciente, con énfasis en los ambientes seguros en salud a través de la gestión de riesgos para evitar el error ${ }^{(12)}$. La JCl es una importante organización no gubernamental americana que evalúa la calidad del servicio de los hospitales en los EE.UU. En Brasil, la JCl recomienda que existan herramientas para medir y monitorear el desempeño de una institución y propone el uso de instrumentos de notificación y análisis crítica sobre las causas de su ocurrencia e implantación de medidas de calidad ${ }^{(12)}$. 
Los Estados Unidos de America lideraron las publicaciones. La cuestión de seguridad del paciente en este país se ha convertido en objeto de interés permanente y se convirtió en ámbito internacional, desde la publicación del informe "Errar es humano" (To Err is Human), elaborado por el Comité para la Calidad del Cuidado a la Salud en America (Committee on Quality of Health Care in América), publicado en $200{ }^{(13)}$. Otra iniciativa importante en el ámbito de la seguridad de los pacientes en este país fue la creación de la Alianza Mundial para la Seguridad del Paciente puesta en marcha en octubre de 2004 por la Organización Mundial de la Salud (OMS) con la misión de coordinar y difundir prácticas de mejora para la seguridad del paciente, en todo el mundo ${ }^{(14)}$.

El Reino Unido ha destacado en segundo lugar con $13,5 \%$ de las publicaciones. Ese país, en los años 90, pasó por un proceso similar a los EE.UU., con un aumento de denuncias de incidentes relacionados con equipos médicos. En 1999 fueron registrados más de 6.600 incidentes, incluyendo 87 muertes y 345 lesiones graves ${ }^{(15)}$. Otra iniciativa de seguridad del paciente en este país se produjo principalmente en 2001 con la creación de la National Patient Safety Agency, que estableció áreas y procedimientos para el monitoreo y acciones de mejora del desempeño de las instituciones de salud con la misión de identificar cuestiones de seguridad del paciente y encontrar soluciones apropiadas ${ }^{(16)}$.

Brasil, en este trabajo, estuvo en $9,4 \%$ de las publicaciones. El país se destaca por la creación de una de las primeras y principales estrategias para la seguridad del paciente, la Agencia Nacional de Vigilancia Sanitaria, agencia del gobierno, que ha trabajado mucho en el campo de la seguridad. Su propósito institucional es promover la protección de salud de la población, mediante el control sanitario de la producción y comercialización de productos y servicios sujetos a la vigilancia sanitaria, incluyendo los ambientes, procesos, insumos y las tecnologías con ellos relacionadas, así como el control de los puertos, aeropuertos y fronteras ${ }^{(17)}$.

En cuanto al enfoque temático de las publicaciones $20,8 \%$ enfocan el error médico. El error médico se define como el resultado de la falta de uso de las medidas de seguridad de los pacientes para evitar todo tipo de eventos adversos o tipos diferentes de eventos, tales como los errores de medicación, errores quirúrgicos, errores de comunicación o errores en el diagnóstico ${ }^{(18)}$. Muchos autores sugieren la implantación de un protocolo y medidas de seguridad para ayudar en la prevención de la ocurrencia de eventos adversos, eliminando dudas y confusiones en relación con la realización de un procedimiento, y facilitando la comunicación entre los miembros del equipo, siendo ciertamente efectiva en la prevención de errores y eventos adversos derivados de la atención recibida ${ }^{(19,20)}$. Dos pesquisidores citan la falta de cultura de seguridad en las instituciones como la raíz del problema del error médico ${ }^{(21,22)}$. Sin embargo, hay necesidad de alinear los planes estratégicos de las organizaciones creando un ambiente propicio para la gestión de riesgos y prevención de eventos adversos con un programa de mejora de la calidad y seguridad del paciente y reducción de los costes derivados de la prestación de los cuidados ${ }^{(23)}$.

La calidad de la atención está presente en 16\% de las publicaciones. Los estudios destacan las relaciones entre las características organizativas y culturales de los hospitales, el impacto de estos en la calidad de los cuidados y el enfoque en resultados proyectados para ayudar en las decisiones políticas, así como los contribuyentes y gestores de los hospitales para comprender los factores y procesos que permiten alcanzar y mantener servicios de alta calidad para sus pacientes ${ }^{(24,25)}$. 
El error de medicación obtuvo 12,3\% de las publicaciones. La mayoría de los estudios sobre este tema se relaciona con errores ocurridos en la preparación y administración por el equipo de enfermería y describen el conocimiento y habilidad en la toma de decisiones sobre todo por los enfermeros en relación a las prácticas seguras durante el proceso y la administración de medicamentos ${ }^{(26)}$. El error de medicación es considerado un error en los pasos del proceso de medicación - requisición, distribución, administración y monitorización del efecto - causando daño o implicando riesgo de dañar al paciente ${ }^{(27)}$. Mejorar la seguridad durante el proceso y la administración de medicamentos es un objetivo mundial visto que contribuye a la alta tasa de eventos adversos, en los hospitales, a menudo son costosos y perjudiciales para los pacientes, profesionales de salud y hospitales. Entre los factores de riesgo en el sistema de medicación están la organización, distribución y embalaje de los medicamentos en la estación de enfermería ${ }^{(28)}$.

Otro estudio examinó la redacción de la prescripción médica en los errores de dosis, ocurridos en unidades de clínicas médicas de cinco hospitales brasileños, y concluyó que la ausencia de la posología en las prescripciones puede favorecer la administración de dosis equivocadas, lo que resulta en un tratamiento ineficaz, comprometiendo la calidad de la atención prestada a los pacientes hospitalizados (26)' De ahí la importancia de la notificación del error de medicación, de la adecuación de la carga de trabajo y del abordaje de estrategias para prevenir los errores de medicación (29). Por otra parte, la identificación adecuada del paciente ha sido considerada necesaria para una administración de medicamentos de manera segura, para evitar que los clientes con nombres similares sean fácilmente confundidos y reciban una dosis inadecuada para su tratamiento ${ }^{(26)}$.

El tema gestión del riesgo ha sido encontrado en $12,3 \%$ de las publicaciones y presupone una serie de acciones (prevención, detección y mitigación) para la prevención y la reducción del riesgo de daños innecesarios para el paciente asociado con el cuidado de la salud ${ }^{(30)}$. En dos estudios la adherencia al cheklist fue citada como un importante método para la gestión de la seguridad en una institución ${ }^{(31,32)}$.

La actitud profesional frente al error/ riesgo fue citada en $11,3 \%$ de las publicaciones. Los estudios indican la necesidad de la opinión de los profesionales para conocer y evaluar los obstáculos y los condicionantes en su práctica clínica, para el pleno desarrollo de la cultura de seguridad en la práctica diaria, así como los elementos positivos que emergen del sistema y que se presentan como aliados para favorecer un cambio organizacional hasta una verdadera cultura de seguridad del paciente ${ }^{(33)}$.

El tema cultura de seguridad alcanzó $8,5 \%$ de las publicaciones y vinculan los procesos de cambio e innovación en instituciones con diferentes culturas organizacionales y factores subyacentes que facilitan o dificultan este proceso. Estos factores incluyen a personas, ideas, padrones de relación y tiempo. Se puede decir que en ciertos ambientes hay una cultura organizacional que favorece el cambio y la innovación y en otros, no. La creación de un ambiente seguro para los pacientes y profesionales requiere un plan estratégico, liderazgo y trabajo en equipo ${ }^{(34)}$.

Los indicadores de calidad obtuvieron 3,8 \% de las publicaciones. Los hospitales son responsables por una parte significativa de la asistencia de salud prestada a los pacientes y la inclusión de indicadores de seguridad en los programas de vigilancia de la calidad en estos sitios contribuye a orientar medidas que promueven la seguridad 
del paciente hospitalizado. En Brasil, hay un conjunto de indicadores desarrollados para este propósito, aunque hay poca investigación sobre el tema, con el fin de contribuir al desarrollo de un conjunto de medidas de seguridad adaptadas a la realidad brasileña. Es importante que los indicadores de seguridad del paciente sean, basados en la mejor evidencia científica, adaptados a la realidad de cada país, para asegurar su viabilidad. Este proceso debe considerar las variaciones culturales de la práctica clínica, la disponibilidad de sistemas de información y la capacidad de los hospitales y sistemas de salud para implementar programas efectivos de vigilancia de la calidad ${ }^{(34)}$.

El tema sobre el liderazgo del enfermero estuvo en 3,8\% de las publicaciones. En estos estudios, los autores presentan a los enfermeros como líderes del equipo, líneas de frente en la seguridad y en la calidad en los procesos y resultados de la atención a la salud. Como tal, los enfermeros son obligados a desarrollar las habilidades necesarias para mejorar la asistencia prestada como una responsabilidad profesional. Como líderes de una organización se convierten en el vínculo primario en el desempeño de la misma; identifican y comunican los valores colectivos, aseguran los recursos para las personas, escuchan la mayoría de las veces, pues son los modeladores y defensores de culturas orientadas al bueno desempeño ${ }^{(35)}$.

El tema de la comunicación estuvo presente en 2,8\% de las publicaciones. Fallas en este segmento fueron citadas como uno de los factores que contribuyen para la mayoría de los casos de daño para el paciente y se produce en niveles inaceptables. Incluso teniendo consciencia de que el trabajo en equipo y la buena comunicación entre el equipo es esencial para la prestación de cuidados de elevada calidad, los procesos y sistemas proyectados para mejorar esa comunicación siguen siendo escasos $^{(36)}$.

Capacitación en servicio alcanzó 2,8\% de las publicaciones. La técnica de gestión de recursos ha sido resaltada como herramienta importante para determinar el costoefectividad de la capacitación en el servicio de salud. Los autores estudiaron la actitud, comportamiento y organización, por medio de la evaluación de barreras y facilitadores para estimular el individuo y el equipo a tener consciencia de las amenazas y peligros para gestionar situaciones de riesgo de manera efectiva. Independiente de evitar los errores, esta técnica puede cooperar para el cambio de la cultura, produciendo un ambiente más seguro ${ }^{(37)}$.

El recurso humano y financiero de la salud estuvo presente en el $2,8 \%$ de las publicaciones. Las personas fueron destacadas como clave para mejorar la calidad y el departamento de gestión de recursos humanos tiene un papel fundamental, dentro de una organización, en relación con las personas y es capaz de proporcionar una inversión financiera para permitir el crecimiento de la institución con calidad. Se resalta la necesidad de una mejora en la cultura organizacional cuya eficacia depende de factores tales como el liderazgo, cultura, creación de conocimiento y aprendizaje, factores que no pueden ser comprados, pero sí construidos en conjunto. La capacidad y el compromiso de los gestores en la implementación de los cambios resultarán en el éxito o fracaso de la organización, lo que significa tener profesionales con un enfoque en la seguridad del paciente y en la calidad de la atención prestada ${ }^{(38)}$.

Registro electrónico estuvo presente en 1,9\% de las publicaciones. Los estudios abordan la importancia de los sistemas de registro electrónicos en salud para un impacto positivo en la seguridad del paciente. Por lo tanto, los profesionales deben 
estar preparados y habilitados para utilizar eficazmente esta herramienta ${ }^{(39)}$.

En cuanto a la normalización de la terminología y conceptos fueron $0,9 \%$ de las publicaciones. Los autores sugerirán una definición comprensible de error médica y seguridad del paciente e incentivarán la normalización de los términos utilizados en cuidados de salud por medio de una revisión sistemática de la literatura, con el objetivo de estandarizar la terminología utilizada en la investigación de la seguridad del paciente, partiendo de las recomendaciones de Organización Mundial de la Salud (OMS) y, el uso de los conceptos de seguridad de los pacientes a nivel internacional aceptables para la clasificación de eventos adversos y casi errores en las instituciones de salud de todo el mundo ${ }^{(40)}$.

\section{CONCLUSIONES}

El tema ha sido investigado de forma continua durante los últimos tres años (20102012 ), con el predominio de la producción de artículos originales (74,5\%), cuyos principales vehículos fueron los periódicos, lo que facilita la difusión del conocimiento.

El predominio de las publicaciones de los EEUU (50,9\%), natural por la gran inversión en investigación en este país, contrasta con la escasez de publicaciones sobre seguridad de los pacientes en Brasil, lo que demuestra la necesidad de estudios brasileños en este segmento.

La preocupación de los estudiosos frente a los errores y sugerencias en la prevención de sus ocurrencias muestran el reconocimiento de la importancia de una atención segura y de calidad como base para la práctica profesional competente.

Los errores ocurridos en la preparación y administración de medicamentos destacan al enfermero como el principal profesional en este campo de trabajo enfatizando la importancia de un equipo cualificado para hacer frente a la práctica de medicación segura. Iniciativas sobre medicación segura fueron lanzadas en todo el mundo, pero aun es necesario investigaciones intervencionistas para la creación de sistemas más seguros y de calidad.

Mejoría institucional en relación a la cultura de seguridad es compromiso de la gestión hospitalaria, cuyos profesionales de salud son esenciales en esta decisión, siendo la administración del hospital la responsable del establecimiento de políticas y procedimientos para la mejora de la calidad y cultura de seguridad en el hospital ${ }^{(41)}$.

Algunos estudios demostrarán la importancia de la implementación de un programa global y multidimensional para evaluar y mejorar la calidad de la atención de salud como una prioridad institucional destinada a la cultura de seguridad con la inversión en el desarrollo de las capacidades locales, en gestión de riesgos y en los sistemas de información que ya existen ${ }^{(34)}$. Es necesario orientar la construcción de programas de vigilancia para garantizar la producción de información para la toma de decisiones y estimular el compromiso de todos con la transparencia en la atención de salud y la responsabilidad con la mejoría de la seguridad.

Este estudio tiene como objetivo despertar la construcción del conocimiento sobre seguridad del paciente y las actitudes de los profesionales en lo que se refiere a la cultura organizacional revelan claramente la falta de comunicación y gestión de riesgos, del registro electrónico eficaz, de la falta de estandarización de la 
terminología relacionada con los eventos adversos, de la preocupación del liderazgo con el crecimiento institucional, con actitudes proactivas frente al error y la necesidad de capacitación en el servicio. Actuar para el alcance de la cultura de seguridad de las organizaciones requiere un cambio del pensamiento, uso de registros adecuados y conocimiento de los errores actuales. El resultado aquí expuesto puede ayudar al desarrollo de nuevas investigaciones sobre el tema, buscando esclarecer lo que sucede en la práctica profesional y así proponer estrategias para evitar la repetición de errores mejorando la cultura organizacional y con eso, la prevención de eventos adversos y la mejoría de la calidad y seguridad de la atención al paciente .

\section{REFERENCIAS BIBLIOGRÁFICAS}

1. World Health Organization (WHO). World Alliance for Patient Safety. Research for patient safety: better knowledge for safer care. 2008; 20. Disponível em: $<$ http://www.who.int/patientsafety/information_centre/documents/ps_research_brochur e_en.pdf>. Acesso em: 08/07 2012.

2. Bonato VL. Gestão de qualidade em saúde: melhorando assistência ao cliente Health quality management: improving support to client Gestión de calidad en salud: mejorando asistencia al cliente. São Paulo. 2011;35(5):319-31.

3. Sabino EA. Cultura organizacional: Percepção e Relação com a Satisfação no Trabalho. Monografia. Universidade de Brasília. 2011.

4. Reiling JG. Creating a culture of patient safety through innovative hospital design. Advances in Patient Safety: From Research to Implementation. Volume 3, AHRQ Publication Nos. $050021 \quad(1-4)$. Feb. 2005. Disponível em: <http://www.ahrq.gov/qual/advances/>. Acesso em 25/08/12.

5.Sorra J. Nieva V. F. Hospital Survey on Patient Safety Culture. AHRQ Publication n. 04-0041. Rockville, MD: Agency for Healthcare Research and Quality. Sept. 2004. Disponível em <http://www.ahrq.gov/qual/hospculture/hospcult.pdf> Acesso em 25/08/12.

6. Filho APG. Universidade federal da bahia cultura e gestão de segurança no trabalho em organizações industriais: uma proposta de modelo organizações industriais: Uma proposta de modelo. tese de doutorado. Salvador, 2011.

7. Kirk S, Parker D, Claridge T, Esmail A, Marshall M: Patient safety culture in primary care: developing a theoretical framework for practical use. Qual Saf Health Care 2007, 16(4):313-20.

8. Chen $\mathrm{C}, \mathrm{Li} \mathrm{H}$. Measuring patient safety culture in Taiwan using the Hospital Survey on Patient Safety Culture (HSOPSC). BMC Health Services Research.2010. 10:152

9. Godoy RSP, Pecanha DLN. Cultura organizacional e processos de inovação: um estudo psicossociológico em empresa de base tecnológica. Bol. - Acad. Paul. Psicol., São

Paulo, $\checkmark$. 29, n.1, jun. 2009.Disponível:<http://pepsic.bvsalud.org/scielo.php?script=sci_arttext\& pid=S1415711X2009000100012\&lng=pt\&nrm=iso $>$. Acesso em 25/08/2012.

10. Mueller SPM, Passos EL. Comunicação cientifica. Universidade de Brasília. Departamento de Ciência da Informação e Documentação. 2000 pg 15-16.

11. Santos RN. Produção científica: por que medir? $O$ que medir? Rev Digit Biblioteconomia Cienc Inform. 2003; 1(1):22-38.

14. Joint Commission on Accreditation of Healthcare Organizations (JCAHO). Improving america's hospitals: a report on quality and safety. Disponivel em http://www.jointcommission.org/assets/1/6/2006 Annual Report.pdf. Acesso em $\underline{12 / 06 / 2012 .}$ 
15. Institute Of Medicine. Medicare: a strategy for quality assurance. Volume I. Washington, D.C.: National Academy Press, 1990. Disponível em: <http://www.nap.edu/catalog/1547.html>. Acesso em 10/07/2012.

13. Joint Commission for Patient Safety. World Alliance for Patient Safety [internet]. 2008 Disponível em: http://www.ccforpatientsafety.org/30730/. Acesso em 25/08/2012.

16. Department of Health. An organisation with a memory: report of an expert group on learning from adverse events in the NHS. London: The Stationery Office; 2000. Disponível

em<http://www.dh.gov.uk/en/Publicationsandstatistics/Publications/PublicationsPolicy AndGuidance/DH_4065083> Acesso em 12/06/2012.

17. National Patient Safety Agency (NPSA). Disponivel em http://www.npsa.nhs.uk/. Acesso em 12/08/2012.

18.ANVISA - www.anvisa.gov.br site da Agência Nacional de Vigilância Sanitária. Disponível em http://www.anvisa.gov.br/servicosaude/hsentinela/areas_diretas.htm> Acesso em 12/09/2012.

19. Suresh GK. Measuring patient safety in neonatology. American journal of perinatology [Internet].2012Jan;29(1):19-26.Availabl from: http://www.ncbi.nlm.nih.gov/pubmed/21879457

20. Reason $\mathrm{J}$ : patient safety, human error, and Swiss cheese. Interview by Karolina Peltomaa and Duncan Neuhauser.. Qual Manag Health Care; 21(1): 59-63, 2012 JanMar.

21. Chevillotte J. Promoting an absolute culture of patient safety. Rev Infirm; (177): 4, 2012 Jan.

23. Robertson N. Improving patient safety: lessons from rock climbing. The clinical teacher [Internet]. $2012 \quad$ Feb;9(1):41-4. Available from: http://www.ncbi.nlm.nih.gov/pubmed/22225892

24. Dodek PM; Wong H; Heyland DK; Cook DJ; Rocker GM; Kutsogiannis DJ; Dale C; Fowler R; Robinson S; Ayas NT. The relationship between organizational culture and family satisfaction in critical care. Crit Care Med; 40(5): 1506-12, 2012 May

25. Caixeiro, FTO. Aplicação do método análise dos modos de falha e seus efeitos (FMEA) para a prospecção de riscos nos cuidados hospitalares no Brasil/ Application of the analysis of failure modes and effects (FMEA) for the exploration risk in the hospital in Brazil.

26. Chera BS; Jackson M; Mazur LM; Adams R; Chang S; Deschesne K; Cullip T; Marks LB. Improving quality of patient care by improving daily practice in radiation oncology. Semin Radiat Oncol; 22(1): 77-85, 2012 Jan.

27. Aranaz JM; Moya C. Patient safety and health care quality.Rev Calid Asist; 26(6): 331-2, 2011 Nov-Dec.

28. Gimenes FRE, Mota MLS, Teixeira TCA, Silva AEBDC, Opitz SP, Cassiani SHDB. Patient safety in drug therapy and the influence of the prescription in dose errors. Revista latino-americana de enfermagem [Internet]. 2010;18(6):1055-61. Available from: http://www.ncbi.nlm.nih.gov/pubmed/21340268

29. Lisby M; Nielsen LP; Brock B; Mainz J. How should medication errors be defined? Development and test of a definition. Scand J Public Health; 40(2): 203-10, 2012 Mar. 30. Raduenz AC, Hoffmann P, Radunz V, Dal Sasso GTM, Maliska ICA, Marck PB. Nursing care and patient safety: visualizing medication organization, storage and distribution with photographic research methods. Revista latino-americana de enfermagem [Internet]. 2010;18(6):1045-54. Available from: http://www.ncbi.nlm.nih.gov/pubmed/21340267

31. Bohomol E, Ramos LH. Erro de medicação: importância da notificação no gerenciamento da segurança do paciente. REBEn. 2007;60(2):32-6. 
32. de Paiva MCMDS, de Paiva SAR, Berti HW. [Adverse events: analysis of a notification instrument used in nursing management]. Revista da Escola de Enfermagem da U S P [Internet]. 2010 Jun;44(2):287-94. Available from: http://www.ncbi.nlm.nih.gov/pubmed/20642037

33. Kasatpibal N; Senaratana W; Chitreecheur J; Chotirosniramit N; Pakvipas P; Junthasopeepun $\mathrm{P}$

Implementation of the World Health Organization surgical safety checklist at a university hospital in Thailand. Surg Infect (Larchmt); 13(1): 50-6, 2012 Feb.

34. Helmiö P; Blomgren K; Takala A; Pauniaho SL; Takala RS; Ikonen TS. Towards better patient safety: WHO Surgical Safety Checklist in otorhinolaryngology. Clin Otolaryngol; 36(3): 242-7, 2011 Jun.

35. Ques A, Montoro C, Gonzalez M. Fortalezas e ameaças em torno da segurança do paciente segundo a opinião dos profissionais de enfermagem ${ }^{1}$. 2010;18(3):54-6.

36. Cadmus E; Brigley P; Pearson M. Safe patient handling: is your facility ready for a culture change? Nurs Manage; 42(11): 12-5, 2011 Nov.

37. Gouvêa CSD, Travassos C. Indicadores de segurança do paciente para hospitais de pacientes agudos: revisão sistemática Patient safety indicators for acute care hospitals : a systematic review. 2010;26(6):1061-78.

38. Julianne M. Nurses create a culture of patient safety: it takes more than projects. Online J Issues Nurs; 16(3): 2, 2011 Sep.

39. Morrison D; Sanders C. Huddling for optimal care outcomes. Nursing; 41(12): 224, 2011 Dec.

40. Kemper PF, de Bruijne M, van Dyck C, Wagner C. Effectiveness of classroom based crew resource management training in the intensive care unit: study design of a controlled trial. BMC health services research [Internet]. BioMed Central Ltd; 2011 Jan $\begin{array}{lllll}\text { [cited } 2012 & \text { Oct } & 3] ; 11(1): 304 . & \text { Available }\end{array}$ from:http://www.pubmedcentral.nih.gov/articlerender.fcgi?artid=3248881\&tool=pmcent rez\&rendertype $=$ abstract

41. Singer SJ, Clark JR. Entrepreneurship, culture, knowledge, and learning: a multispective view of patient safety improvement. Health care management review [Internet]. 2011 [cited 2012 Oct 3];36(4):299-300; discussion 303-5. Available from: http://www.ncbi.nlm.nih.gov/pubmed/21712719

42. Metzger J; Welebob E; Bates DW; Lipsitz S; Classen DC Mixed results in the safety performance of computerized physician order entry. Health Aff (Millwood); 29(4): 655-63, 2010 Apr.

43. Keriel-Gascou M; Figon S; Letrilliart L; Chaneliére M; Colin C. Presse Med; 40(11): e499-505, 2011 Nov.

44. Zohar D, Gazit TO: Transformational leadership and group interaction as climate antecedents: A social network analysis. J Appl Psychol 2008, 93:744-757.

ISSN 1695-6141

(c) COPYRIGHT Servicio de Publicaciones - Universidad de Murcia 
